\title{
Evaluation of Matrix-Assisted Laser Desorption/ Ionization Time-of-Flight Mass Spectrometry in Identification of Nontuberculous Mycobacteria
}

\author{
Ivana Marekovića,b Zrinka Bošnjak ${ }^{a, b}$ Marko Jakopovića, ${ }^{a}$ Zagorka Boras ${ }^{c}$ \\ Mateja Jankovića, c Sanja Popović-Grle ${ }^{a, c}$ \\ ${ }^{a}$ School of Medicine, University of Zagreb, and ${ }^{b}$ Department of Clinical and Molecular Microbiology and ${ }^{\mathrm{C} C l i n i c}$ for \\ Pulmonary Diseases Jordanovac, University Hospital Center Zagreb, Zagreb, Croatia
}

\section{Key Words}

Bacterial infection - Respiratory tract infection - Respiratory

tract pathogen - Nontuberculous mycobacteria $\cdot$ MALDI-

TOF MS · Identification

\begin{abstract}
Background/Aims: Species-level identification of nontuberculous mycobacteria (NTM) is important in making decisions about the necessity and choice of antimicrobial treatment. The reason is predictable clinical significance and the susceptibility profile of specific NTM species. Matrix-assisted laser desorption/ionization time-of-flight mass spectrometry (MALDI-TOF MS) is recognized as a diagnostic tool for routine identification of bacteria and yeasts in the clinical laboratory based on protein fingerprint analysis. The aim of the study was to evaluate MALDI-TOF MS in the identification of NTM. Methods: A total of 25 NTM isolates from liquid cultures were identified with both polymerase chain reaction (PCR)-based hybridization assay and MALDI-TOF MS at the University Hospital Center Zagreb. Results: PCR-based hybridization assay identified $96 \%$ (24/25) and MALDI-TOF MS $80 \%(20 / 25)$ of tested NTM isolates. Five isolates with no reliable MALDI-TOF MS identification belonged to the Mycobacterium avium-intracellulare complex. Seventy percent (14/20)
\end{abstract}

of NTM isolates successfully identified with MALDI-TOF MS had a score higher than 2.0, indicating reliable species identification. Conclusion: MALDI-TOF MS is a promising tool for the identification of NTM. With a further improvement of the protein extraction protocol, especially regarding the M. avium-intracellulare complex, MALDI-TOF MS could be an additional standard method for identification of NTM.

(c) 2016 S. Karger AG, Basel

\section{Introduction}

Nontuberculous mycobacteria (NTM) are attracting growing attention due to the increase in the isolation frequency, especially in the countries with a declining tuberculosis incidence [1]. Clinical diseases NTM can cause a range of problems from the most frequent pulmonary disease to lymphadenitis in children, skin disease and disseminated infections in immunocompromised patients [2]. Species-level identification of NTM is important because it can guide the clinician's decision about the necessity and choice of antimicrobial treatment due to predictable clinical significance and susceptibility profile of specific NTM species [3]. Biochemical tests previously used for identification are slow and surpassed by still techni-

\section{KARGER}

E-Mail karger@karger.com

www.karger.com/che
(C) 2016 S. Karger AG, Basel

0009-3157/16/0614-0167\$39.50/0
Ivana Mareković

School of Medicine, University of Zagreb

Kišpatićeva 12

HR-10 000 Zagreb (Croatia)

E-Mail ivanamarekovic@yahoo.com 
Table 1. Identification of NTM isolates by MALDI-TOF MS and PCR-based hybridization assay

\begin{tabular}{lll}
\hline PCR-based hybridization & \multicolumn{2}{l}{ MALDI-TOF MS identification } \\
\cline { 2 - 3 } assay identification & species & score \\
\hline M. abscessus & M. abscessus & 2.208 \\
M. gordonae & M. gordonae & 1.887 \\
M. xenopi & M. xenopi & 1.852 \\
M. avium & no reliable identification & - \\
M. avium & M. avium & 2.009 \\
M. fortuitum & M. fortuitum & 2.370 \\
M. avium & M. avium & 2.036 \\
M. fortuitum & M. fortuitum & 2.465 \\
M. fortuitum & M. fortuitum & 2.308 \\
M. avium & M. avium & 1.906 \\
M. fortuitum & M. fortuitum & 2.256 \\
M. fortuitum & M. fortuitum & 2.411 \\
M. fortuitum & M. fortuitum & 2.384 \\
M. avium & M. avium & 1.978 \\
M. avium & M. avium & 2.252 \\
Negative & M. fortuitum & 2.365 \\
M. avium & M. avium & 2.059 \\
M. xenopi & M. xenopi & 2.187 \\
M. gordonae & M. gordonae & 1.466 \\
M. intracellulare & no reliable identification & - \\
M. avium & M. avium & 1.805 \\
M. intracellulare & no reliable identification & - \\
M. intracellulare & no reliable identification & - \\
M. gordonae & M. gordonae & 2.071 \\
M. intracellulare & no reliable identification & - \\
\hline & & \\
& &
\end{tabular}

cally cumbersome and relatively expensive molecular methods. Polymerase chain reaction (PCR)-based hybridization assays and gene sequencing are now routinely used in laboratories in developed countries [2, 4]. A new tool for species identification of NTM is matrix-assisted laser desorption/ionization time-of-flight mass spectrometry (MALDI-TOF MS) based on unique spectral fingerprints produced by extracted proteins $[4,5]$. The aim of this study was to evaluate the use of MALDITOF MS in the identification of NTM.

\section{Methods}

A total of 25 NTM isolates collected between January and October 2014 at the Department of Clinical and Molecular Microbiology, University Hospital Center Zagreb, Zagreb, Croatia, were tested. The NTM strains were primarily isolated on solid medium (Löwenstein-Jensen). For each isolate a fresh subculture in Mycobacterium Growth Indicator Tube (MGIT) culture bottles incubated in BACTEC ${ }^{\circledR}$ MGITTM 960 System (Becton, Dickinson and Company, Sparks, Nev., USA) was used and identification was done with both, PCR-based hybridization assay and MALDI-TOF MS. For MALDI-TOF MS analysis a protein extraction was performed according to the manufacturer's instructions as follows. A $1.5-\mathrm{ml}$ aliquot was removed from the MGIT culture bottle and centrifuged for $2 \mathrm{~min}$ at 14,000 $\mathrm{rpm}$. The supernatant was removed from the pellet and $500 \mu \mathrm{l}$ of deionized water was added to the pellet and centrifuged for $2 \mathrm{~min}$ at 14,000 rpm. The supernatant was then removed and the pellet was suspended in $300 \mu$ of deionized water before $900 \mu \mathrm{l}$ of $100 \%$ ethanol was added and mixed thoroughly. For inactivation and reducing the number of viable cells after adding of ethanol the sample was incubated for $10 \mathrm{~min}$. The ethanol suspension was centrifuged for $2 \mathrm{~min}$ at 14,000 rpm. The supernatant was then removed and the pellet was suspended in 500 $\mu \mathrm{l}$ of deionized water and centrifuged for $2 \mathrm{~min}$ at 14,000 rpm. The supernatant was removed and the pellet was suspended in $50 \mu \mathrm{l}$ of deionized water and then put in a heat block at $95^{\circ} \mathrm{C}$ for $30 \mathrm{~min}$ to inactivate the organisms. After heat inactivation, the suspension was allowed to cool to room temperature before adding 1,200 $\mu \mathrm{l}$ of $100 \%$ ethanol precooled in a freezer at $-18^{\circ} \mathrm{C}$. The suspension was then vortexed and centrifuged for $2 \mathrm{~min}$ at $14,000 \mathrm{rpm}$. The supernatant was removed, and the pellet was centrifuged again and the residual ethanol was carefully removed by pipetting. After the pellet was completely dry, $25 \mu \mathrm{l}$ of acetonitrile and $0.5 \mathrm{~mm}$ zirconia/ silica beads on the tip of a small spatula were added. The sample was then vortexed for $1 \mathrm{~min}$ to disrupt any aggregates, and $25 \mu \mathrm{l}$ of $70 \%$ formic acid was added and mixed by vortexing for $5 \mathrm{~s}$ and centrifuged for $2 \mathrm{~min}$ at 14,000 rpm. A 1- $\mu$ l portion of supernatant was added on an MALDI target and overlayed with $1 \mu$ l of matrix solution after drying. All samples with no peaks found were submitted to re-extraction. In addition, each extracted sample was deposited twice on the steel target plate and the better score was used for the study purpose.

The extract was analyzed with MALDI Biotyper 3.1 (Bruker Daltonik GmbH, Bremen, Germany) and the spectra obtained were compared to the Mycobacteria Library 1.0 that contains 173 spectra representing 94 mycobacterial species. The Bruker Bacterial Test Standard was used for calibration. Protein profiles with a mass-to-charge ratio $(\mathrm{m} / \mathrm{z})$ of 2,000-20,000 Da were produced based on a laser frequency of $60 \mathrm{~Hz}, 240$ laser shots of each spectrum and 40 shot steps used.

Confidence scores greater than 2.0 were considered secure species identification, scores of 1.7-2.0 were considered intermediate identification, and scores of less than 1.7 were considered unreliable identification. For PCR-based hybridization assay (GenoType Mycobacterium CM; Hain Lifescience, Nehren, Germany) DNA extraction protocol with heat inactivation and ultrasonic bath according to the manufacturer's instructions was used.

\section{Results and Discussion}

PCR-based hybridization assay identified 96\% (24/25) of tested NTM isolates which included Mycobacterium avium $(\mathrm{n}=8), M$. fortuitum $(\mathrm{n}=6), M$. intracellulare $(\mathrm{n}=4), M$. gordonae $(\mathrm{n}=3), M$. xenopi $(\mathrm{n}=2)$, and $M$. abscessus $(\mathrm{n}=1)$ (table 1$)$. Analysis of the same isolates by MALDI-TOF MS identified 80\% (20/25) of tested NTM 
isolates. Five isolates in which identification was not successful included $M$. intracellulare $(\mathrm{n}=4)$ and $M$. avium $(n=1)$. Seventy percent $(14 / 20)$ of isolates successfully identified with MALDI-TOF MS had a score higher than $2.0,25 \%(5 / 20)$ a score ranging from 1.7 to 2.0 , and only $5 \%(1 / 20)$ had a score less than 1.7 (table 2). Out of 24 isolates identified with the PCR hybridization method, $79.2 \%$ (19/24) were also identified with the MALDI-TOF MS as a same mycobacterial species.

At the moment MALDI-TOF MS is recognized as a very simple and fast method with significantly lower consumable costs suitable for routine identification of bacteria and yeasts in the clinical laboratory $[6,7]$. However, the critical point for accurate MALDI-TOF MS identification of NTM isolates is a protein extraction protocol [8]. Direct smearing and simple extraction methods used for other bacteria and yeasts are not sufficient for NTM because of the genus's hardy cell envelope and tendency to form clumps which have to be dispersed during the extraction procedure [9]. Therefore, the majority of successful mycobacteria protein extraction protocols use glass or zirconia-silica beads which yield high-quality spectra and lead to reproducible identifications at the species level. Furthermore, the use of heat in the protocol inactivates mycobacteria and minimizes the exposure of laboratory workers to viable mycobacteria [10].

It is still not clear which medium, liquid or solid, it is better select as a source of NTM isolate for MALDI-TOF MS identification. Some authors had a higher identification rate from solid medium compared to MGIT liquid medium, a possible explanation being the lower number of bacteria obtained in the liquid medium [11]. The authors achieving a better identification rate on liquid media employed another type of liquid media than MGIT in combination with an optimized extraction protocol using formic acid and acetonitrile in addition to physical disruption using silica beads, and, finally, they inoculated liquid medium with subculture from solid medium in order to have a more reproducible biomass [8]. In the present study, MALDI-TOF MS identification was also done from subcultures, although in MGIT culture bottles, an identical extraction procedure was used as in the study mentioned. A high identification rate in our study indicates that the use of a subculture in liquid medium in combination with optimized extraction procedure can make an MGIT liquid medium a favorable source of NTM isolates for identification by MALDI-TOF MS.

Incubation time may also influence the quality of the extract, with some authors suggesting better results for older cultures [11]. In our study, 8 out of a total of 25 iso-

Identification of Nontuberculous

Mycobacteria
Table 2. Scores achieved with MALDI-TOF MS identification of NTM

\begin{tabular}{|c|c|c|c|c|}
\hline \multirow[t]{2}{*}{ NTM isolate } & \multicolumn{3}{|c|}{$\begin{array}{l}\text { MALDI-TOF MS } \\
\text { score, } \mathrm{n}\end{array}$} & \multirow{2}{*}{$\begin{array}{l}\text { No reliable } \\
\text { identification with } \\
\text { MALDI-TOF MS, n }\end{array}$} \\
\hline & $<1.7$ & $1.7-2.0$ & $>2.0$ & \\
\hline M. avium $(\mathrm{n}=8)$ & 0 & 3 & 4 & 1 \\
\hline M. fortuitum $(\mathrm{n}=7)$ & 0 & 0 & 7 & 0 \\
\hline M. intracellulare $(n=4)$ & 0 & 0 & 0 & 4 \\
\hline M. gordonae $(\mathrm{n}=3)$ & 1 & 1 & 1 & 0 \\
\hline M. xenopi $(\mathrm{n}=2)$ & 0 & 1 & 1 & 0 \\
\hline M. abscessus $(\mathrm{n}=1)$ & 0 & 0 & 1 & 0 \\
\hline
\end{tabular}

lates $(32.0 \%)$ were rapid growers represented by $M$. fortuitum and M. abscessus. All of them gain the identification score greater than 2.0 showing excellent species identification. On the other hand, all the NTM isolates with an identification score less than 2.0 were slow growers.

There was a high level of agreement between MALDITOF MS identification and the PCR-based hybridization assay in terms of NTM species identified. In other studies, misidentification and lack of correlation with standard methods were mainly attributed to phylogenetically close species such as $M$. avium and $M$. intracellulare and rapidgrowth NTM $[8,11]$.

If we consider the PCR-hybridization method as one of the standards for the identification of NTM, the correlation between the standard method and MALDI-TOF in our study was $79.2 \%$. Similar results were described in other studies using a similar extraction protocol $[8,12-$ 15]. It should be emphasized that our study is limited by the small number of NTM isolates. Furthermore, only six of the most frequent clinical NTM species were analyzed and several studies revealed that limitations of MALDITOF MS for NTM species identification are associated with less frequent species $[8,12,14]$. Furthermore, $70 \%$ of isolates successfully identified with MALDI-TOF MS had a score higher than 2.0 indicating secure species identification.

For one isolate of $M$. gordonae the identification score was only 1.466. Although the criteria categorize this score as not a reliable identification, due to the concordant PCR-based hybridization method, this identification was considered correct. Some authors suggest decreasing MALDI-TOF MS species-level identification score thresholds in order to resolve more mycobacterial identifications [16-18]. By lowering the MALDI score for the species level from 2 to 1.9 , some of the authors were able 
to resolve $97.2 \%$ mycobacterial identifications to the species level [16]. Furthermore, decreasing the species-level threshold score, the performance of solid and liquid media was similar [16]. However, the optimizing of extraction protocol to improve identification should still be a cornerstone for more successful MALDI-TOF MS identification of NTM.

However, $M$. intracellulare isolates in our study were successfully identified only with PCR-based hybridization methods while MALDI-TOF MS gave no reliable identification. Whether M. intracellulare has some characteristics which make this species difficult to identify with MALDITOF MS remains unclear. One of the possible explanations proposed in recent studies is the presence of glyco- peptidolipids in the cell wall of several mycobacterial species including the M. avium-intracellulare complex [19]. Glycopeptidolipids may be responsible for some characteristics in mycobacteria such as biofilm formation. This could prevent protein extraction reagents from penetrating the cell wall in these NTM species and resulting in no reliable MALDI-TOF MS identification [9].

In conclusion, MALDI-TOF MS is a promising diagnostic tool for identification of NTM isolates. Its simplicity, speed and further improvement of protein extraction protocol could make this method the future standard method for the identification of NTM in routine microbiological diagnostics.

\section{References}

1 Janković M, Samaržija M, Sabol I, Jakopović M, Katalinić Janković V, Žmak L, Tičac B, Marušić A, Obrovac M, van Ingen J: Geographical distribution and clinical relevance of non-tuberculous mycobacteria in Croatia. Int J Tuberc Lung Dis 2013;17:836-841.

2 van Ingen J: Microbiological diagnosis on nontuberculous mycobacterial pulmonary disease. Clin Chest Med 2015;36:43-54.

3 Griffith DE, Aksamit T, Brown-Elliott BA, Catanzaro A, Daley C, Gordin F, Holland SM, Horsburgh R, Huitt G, Iademarco MF, Iseman M, Olivier K, Ruoss S, von Reyn CF, Wallace RJ, Winthrop K: An Official ATS/ IDSA Statement: Diagnosis, Treatment, and Prevention of Nontuberculous Mycobacterial Diseases. Am J Respir Crit Care Med 2007; 175:367-416.

4 van Ingen J: Diagnosis of nontuberculous mycobacteria. Semin Respir Crit Care Med 2013; 34:103-109.

5 Randell P: It's a MALDI but it's a goodie: MALDI-TOF mass spectrometry for microbial identification. Thorax 2014;69:776-778.

6 Bizzini A, Greub G: Matrix assisted laser desorption/ionization time-of-flight mass spectrometry, a revolution in clinical microbial identification. Clin Microbiol Infect 2010;16: 1614-1619.

7 Bošnjak Z, Plečko V, Budimir A, Mareković I, Bedenić B: First report of NDM-1-producing Acinetobacter guillouiae. Chemotherapy 2014;60:250-252.

8 Buchan BW, Riebe KM, Timke M, Kostrzewa M, Ledeboer N: Comparison of MALDI-TOF MS with HPLC and nucleic acid sequencing for the identification of Mycobacterium species in cultures using solid medium and broth. Am J Clin Pathol 2014;141:25-34.
9 Saleeb PG, Drake SK, Murray PR, Zelazny M: Identification of mycobacteria in solid-culture media by matrix-assisted laser desorption ionization-time of flight mass spectrometry. J Clin Microbiol 2011;49:1790-1794.

10 Khechine AE, Couderc C, Flaudrops C, Raoult D, Drancourt M: Matrix-assisted laser desorption/ionization time-of-flight mass spectrometry identification of mycobacteria in routine clinical practice. PLoS One 2011; 6:e24720.

11 Lotz A, Ferroni A, Beretti, Dauphin B, Carbonelle E, Guet-Revillet H, Veziris N, Heym B, Jarlier V, Gaillard JL, Pierre-Audigier C, Frapy E, Berche P, Nassif X, Bille E: Rapid identification of mycobacterial whole cells in solid and liquid culture media by matrixassisted laser desorption ionization-time of flight mass spectrometry. J Clin Microbiol 2010;48:4481-4486.

12 Tudó G, Monté MR, Vergara A, Lopez A, Hurtado JC, Ferrer-Navarro M, Vila J, Gonzalez-Martin J: Implementation of MALDITOF MS technology for the identification of clinical isolates of Mycobacterium spp. in mycobacterial diagnosis. Eur J Clin Microbiol Infect Dis 2015;34:1527-1532.

13 Quinlan P, Phelan E, Doyle M: Matrix-assisted laser desorption/ionization time-of-flight (MALDI-TOF) mass spectrometry (MS) for the identification of mycobacteria from MBBacT ALERT 3D liquid cultures and Lowenstein-Jensen (LJ) solid cultures. J Clin Pathol 2015;68:229-235.
14 Mediavilla-Gradolph MC, De Toro-Peinado I, Bermudez-Ruiz MP, Garcia-Martinez Mde L, Ortega-Torres M, Montiel Quezel-Guerraz N, Palop-Borras B: Use of MALDI-TOF MS for identification of nontuberculous mycobacterium species isolated from clinical specimens. Biomed Res Int 2015;2015:854078.

15 Rodriguez-Sanchez B, Ruiz-Serrano MJ, Marin M, et al: Evaluation of matrix-assisted laser desorption ionization-time of flight mass spectrometry for identification of nontuberculous mycobacteria from clinical isolates. J Clin Microbiol 2015;53:2737-2740.

16 Balada-Llasat JM, Kambij K, Pancholi P: Identification of mycobacteria from solid and liquid media by matrix-assisted laser desorption ionization-time of flight mass spectrometry in the clinical laboratory. J Clin Microbiol 2013;51:2875-2879.

17 Wilen CB, McMullen AR, Burnham CA: Comparison of sample preparation methods, instrumentation platforms, and contemporary commercial databases for identification of clinically relevant mycobacteria by matrixassisted laser desorption ionization-time of flight mass spectrometry. J Clin Microbiol 2015;53:2308-2315.

18 Mather CA, Rivera SF, Butler-Wu SM: Comparison of the Bruker Biotyper and Vitek MS matrix-assisted laser desorption ionizationtime of flight mass spectrometry systems for identification of mycobacteria using simplified protein extraction protocols. J Clin $\mathrm{Mi}$ crobiol 2014;52:130-138.

19 Tatham E, Sundaram Chavadi S, Mohandas P, Edupuganti UR, Angala SK, Chatterjee D, Quadri LE: Production of mycobacterial cell wall glycopeptidolipids requires a member of MbtH-like protein family. BMC Microbiol 2012;12:118. 